\title{
IDAC computer interfacing for an undergraduate learning laboratory
}

\author{
JOHN W. KULIG \\ Plymouth State College, Plymouth, New Hampshire
}

\begin{abstract}
A hardware and software interface system from International Data and Acquisition and Control, Inc. (IDAC) is reviewed. The IDAC system comes with spreadsheet software compatible for either Macintosh or IBM microcomputers. I prefer to use the IDAC hardware with my own software written in TrueBasic and spreadsheet macros. The entire system is easy to use and is currently in use for our undergraduate Learning Laboratory.
\end{abstract}

Despite the wide array of hardware and software available for interfacing laboratory equipment to computers for on-line data collection (Skinner boxes, physiological recordings, observational data, and the like), a standard hardware and software protocol has not emerged, as is evidenced by the number of recent articles on specialized interface systems (for a discussion, see Schneider, 1991). Complete turnkey systems are available, but they are expensive and are not suited for all purposes. Choices among these systems narrow when Macintosh microcomputers are used instead of IBM. Such was our situation when we searched for a general-purpose, modestly priced interface system for the Macintosh to control operant chambers in our undergraduate Learning Laboratory. Few ready-to-run interfaces exist for Macintosh-based laboratory interfaces. MacLab (Costin, 1988) is a Macintosh-based laboratory tool that requires no programming, but only for tasks in which data are input through the keyboard, as in human cognitive research. A sophisticated Macintosh laboratory system is possible with National Instrument's (Austin, TX) NuBus boards controlled with LabVIEW software (see Bates, 1991). These systems can be expensive. While our immediate need was to computerize an undergraduate learning laboratory, we wanted a relatively powerful system that required little technical background and could be used for other tasks.

We opted for a hardware/software system from IDAC (International Data Acquisition and Control Inc., P.O. Box 397, Amherst, NH 03031). The cost of each unit was less than $\$ 2,000$ for hardware and software, and the system is compatible with Macintosh and IBM. The IDAC system is a general-purpose digital and analog interface with a variety of optional peripheral equipment, such as termination relay panels for digital signals and thermocoupler and strain gauges for analog signals. This

Copies of the complete TrueBasic and Excel macro programs, or copies of this article may be obtained by contacting the author at the Department of Psychology, Plymouth State College, Plymouth, NH 03264 (e-mail: johnk@psc.plymouth.edu). article will summarize our use of the digital I/O functions. Several years ago Hatten and Hatten (1989) described the use of an IDAC system to control several operant chambers from one Macintosh. Their article described an early version of the IDAC system that used separate onboard counters and timers that could monitor several channels simultaneously. IDAC no longer builds this option into the system, so we use a separate Macintosh and IDAC unit for each operant chamber. The software from IDAC appears to have changed since the time of the Hattens' article as well.

\section{The IDAC Hardware}

IDAC sells several interfaces, which differ only in the number of channels available. We use the IDAC 1000 series; a 2000 series is available at a higher cost. The IDAC 1000 unit is a Z-80-based minicomputer with $8 \mathrm{~K}$ of RAM, outfitted for analog and digital I/O. Communication between the IDAC unit and the computer occurs through a standard serial cable. The IDAC unit itself measures $32 \mathrm{~cm}$ wide, $43 \mathrm{~cm}$ deep, and $5 \mathrm{~cm}$ high.

The IDAC 1000 permits 2 bytes of digital input and/or output. The 16 bits can be all input, all output, or half of each. The digital termination panel has 16 optically isolated relays that accept standard $24-\mathrm{V}$ input signals. The output relays can route direct or alternating current.

The analog side of the IDAC 1000 offers 12-bit ana$\log$ resolution (.025\% total error), 8 single-ended channels or 4 differential channels, and 3600 analog-to-digital conversions per second. The input signal range is $\pm 10 \mathrm{~V}$. Thermocoupling and strain gauge peripherals are available as optional hardware. We have run IDAC hardware and software on the older Macintosh Plus and SE models, so our learning laboratory has equipped itself by collecting discarded older computers.

\section{The IDAC Software}

Both analog and digital $\mathrm{I} / \mathrm{O}$ are controllable from IDAC software (MaControl for Macintosh, IControl for IBM). The software is spreadsheet based. We use Microsoft Excel, but Multiplan works as well. An application 
spreadsheet directs the sequences of data collection and control of experiments (IDAC provides application spreadsheet templates), and results are written to a report or log spreadsheet. Controlling experiments and collecting data is a simple matter of filling into the appropriate column of the application spreadsheet, which has dedicated functions for different columns. The software can run in a simple acquisition mode, collecting and summarizing data from one source repeatedly, or a control mode, in which input values or passage of time direct sequence steps.

Consider the application spreadsheet for an operant fixed ratio 4 (FR 4) schedule in Figure 1. Column B contains user-entered sequence numbers, specifying the order of execution of steps. Column A has user-entered channel numbers that correspond to I/O functions such as barpress inputs and reward output. In this example, Channel 08 represents the barpress and Channel 00 the pellet dispenser of an operant chamber. Sequence 1 on line 39 says sample the barpress channel continuously (C) to a criterion of 4 , at which time branch to Sequence 2 to pulse $(\mathrm{P})$ the pellet dispenser.

MaControl creates a LOG output file at run time. Figure 2 contains typical output from our FR 4 experiment in the form of a running log that notes the sequence number and type, mean input value divided by number of pulses (this has little meaning for digital $\mathrm{I} / \mathrm{O}$ ), and the start and end time of all input sequences in ticks of $1 / 60 \mathrm{sec}$. Notice that the times are of the first and last response of a sequence, not every response. So the times in columns $\mathrm{H}$ and I are times of the first and fourth response of the sequence. To time every barpress in the FR 4 schedule, one would write four sequences, one for each response. The IDAC software does not convert time ticks to responses per unit time.

Figures 3 and 4 describe a fixed interval 2-min schedule and log summary. The branching is controlled by timing sequences in column $W$ that specify two sequences ( 3 and 4 ) of 1 min each, after which Sequence 1 waits for a single barpress and Sequence 2 delivers a food pellet. The log report for such time-based controls returns the number of barpresses in each of the 1 -min sequences. Thus, in column D of Figure 4, we have 10 and 13 barpresses for the first two 1-min intervals, 14 and 11 for the next two 1-min intervals, and so forth. On columns not shown in the figure, the software also returns the time of first and last responses of a sequence. For greater precision in tracking the data, I could have made the successive intervals less than $1 \mathrm{~min}$ at the expense of a longer application spreadsheet and log report. In the $\log$, every sequence generates a new output line. Other columns in the $\log$ file are for analog signals. Columns $E$ and $F$, for instance, monitor the number and standard deviations of analog readings.

\begin{tabular}{|c|c|c|c|c|c|c|c|c|c|c|c|c|}
\hline & A & B & C & D & K & $\mathbf{L}$ & s & $T$ & $U$ & V & W & $x$ \\
\hline 1 & \multicolumn{12}{|c|}{ MACONTROL - APPLICATION TEMPLATE } \\
\hline 2 & & $\mathbf{S}$ & & & $\#$ & Dur & & & & & & \\
\hline & Exintest & asions & & & 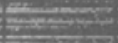 & & & & & & & \\
\hline 22 & & S & & & & & & & & & & \\
\hline 23 & ANALOC & $\mathbf{E}$ & Outp & & & & & & Bran & ch & & \\
\hline 24 & OUTPUT & Q & Val & & & & & & to: & & & \\
\hline 25 & $\mathrm{CH} \# 00$ & & & & & & & & & & & \\
\hline 26 & $\mathrm{CH} \# 01$ & & & & & & & & & & & \\
\hline 27 & & $\mathrm{~S}$ & & & $\#$ & Dur & & & & & & \\
\hline 28 & & E & & On/Of1 & of & betw & & & & & Timer & Branch \\
\hline 29 & DIGITAL & Q & $1 / 0$ & $(1,0)$ & Samp & Samp & Criteria & $\mathrm{BOL}$ & $=$ & $\mathrm{BOH}$ & (minutes & to: \\
\hline 30 & & & & & & & & & & & & \\
\hline 31 & $\mathrm{CH} \# 00$ & 2 & 0 & $P$ & & & & & 1 & & & \\
\hline 32 & $\mathrm{CH} \# 01$ & & & & & & & & & & & \\
\hline 33 & $\mathrm{CH} \# 02$ & & & & & & & & & & & \\
\hline 34 & $\mathrm{CH} \# 03$ & & & & & & & & & & & \\
\hline 35 & $\mathrm{CH} \# 04$ & & & & & & & & & & & \\
\hline 36 & $\mathrm{CH} \# 05$ & & & & & & & & & & & \\
\hline 37 & $\mathrm{CH} \# 06$ & & & & & & & & & & & \\
\hline 38 & $\mathrm{CH} \# 07$ & & & & & & & & & & & \\
\hline 39 & $\mathrm{CH} \# 08$ & 1 & I & & C & & 4 & & 2 & & & \\
\hline 40 & $\mathrm{CH} \# 09$ & & & & & & & & & & & \\
\hline 41 & $\mathrm{CH} \# 10$ & & & & & & & & & & & \\
\hline 42 & $\mathrm{CH} \# 11$ & & & & & & & & & & & \\
\hline 43 & $\mathrm{CH} \# 12$ & & & & & & & & & & & \\
\hline 44 & $\mathrm{CH} \# 13$ & & & & & & & & & & & \\
\hline 45 & $\mathrm{CH} \# 14$ & & & & & & & & & & & \\
\hline 46 & $\mathrm{CH} \# 15$ & & & & & & & & & & & \\
\hline
\end{tabular}

Figure 1. IDAC's application template for an FR 4 operant schedule. 


\begin{tabular}{|c|c|c|c|c|c|c|c|c|c|}
\hline & A & $\mathbf{B}$ & C & D & $\bar{E}$ & $\bar{F}$ & $\mathbf{G}$ & $\mathrm{H}$ & 1 \\
\hline 1 & & $\# \# *$ & 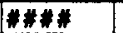 & & & & & & \\
\hline 2 & & & & & & & & & \\
\hline 3 & START D & $\# \# \#$ & START T: & 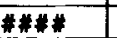 & 38520 & & & & \\
\hline 4 & TICS $(1 / 6)$ & SINCES & 6482 & & & & & & \\
\hline 5 & & & & & & & & & \\
\hline 6 & PROCESS & 1 & & & & & & & \\
\hline 7 & & & & & & & & & \\
\hline 8 & $\widehat{\mathrm{CH}_{*}}$ & SEQ & SEO & MEAN/ & NUMBCR & STANDAF & LAST & TIME OF FIRST & $\overline{E D}$ \\
\hline 9 & & NM & TYPE & PULSES & READNEA & DEVIATIO & VALUE & DIG PULSE(TIC) & TIME(TICS) \\
\hline 10 & $0-8$ & 1 & DI & 3.5 & & & & 7354 & 7645 \\
\hline 11 & 0.0 & 2 & $D$ & & & & & & 7651 \\
\hline 12 & $0-8$ & 1 & D & 3.5 & & & & 7709 & 7911 \\
\hline 13 & $0-0$ & 2 & $\infty$ & & & & & & 7917 \\
\hline 14 & $0-8$ & 1. & D & 3.5 & & & & 7967 & 8360 \\
\hline 15 & 10.0 & 2 & $D$ & & & & & & 8366 \\
\hline 16 & $0-8$ & 1 & d & 3.5 & & & & 8392 & 8619 \\
\hline 17 & $0-0$ & 2 & $\infty$ & & & & & & 8626 \\
\hline 18 & 0.8 & 1 & D & 3.5 & & & & 8775 & 9002 \\
\hline 19 & $0-0$ & 2 & $\infty$ & & & & & & 9008 \\
\hline 20 & $0-8$ & 1 & D & 3.5 & & & & 9107 & 9631 \\
\hline 21 & $0-0$ & 2 & $\infty$ & & & & & & 9637 \\
\hline 22 & $0-8$ & 1 & D & 3.5 & & & & 9682 & 10014 \\
\hline 23 & $0-0$ & 2 & $\infty$ & & & & & & 10021 \\
\hline 24 & $0-8$ & 1 & D & 3.5 & & & & 11123 & 11456 \\
\hline 25 & $0-0$ & 2 & $\infty$ & & & & & & 11462 \\
\hline $2 B$ & $0-8$ & 1 & DI & 3.5 & & & & 11556 & 12045 \\
\hline 27 & 0.0 & 2 & $\infty$ & & & & & & 12051 \\
\hline 28 & $0-8$ & 1 & D & 3.5 & & & & 12111 & 12620 \\
\hline 29 & $0-0$ & 2 & $D$ & & & & & & 12626 \\
\hline
\end{tabular}

Figure 2. IDAC's LOG report of the FR 4 experiment.

MaControl has several drawbacks when used for operant learning situations. First, only the times of the first and last inputs of a sequence are returned. To time every barpress in a ratio schedule, one must program separate sequences for every response. An FR 50 would then require 50 sequence lines in the application spreadsheet. For interval or time-based sequences, there is no way to track the time of each response. Second, it is difficult to program randomness into the application spreadsheet, as would be required, for example, for variable schedules. Excel's RAND function delivers a number between 0 and 1 , but the resultant random value is fixed until the spreadsheet is closed and reopened, making this function useless here. Third, the software cannot ordinarily control more than one operant chamber per interface unit, nor can it control a concurrent schedule with two response alternatives. The reason is that the application spreadsheet permits display of only one sequence at a time, so that only one thing at a time can be monitored. On the other hand, numerous other features of MaControl are useful for other situations; one such feature is the option of sampling only at specified times. The software seems best suited for situations in which one needs summaries of large amounts of continuous data. I now write my own software for the interfacing. For our undergraduate laboratory, my control software is written in TrueBasic (Lebanon, NH), and I summarize the data with Microsoft Excel macro programs. The use of Basic and Excel makes modifying the programs easy, but it is done at the expense of speed.

\section{IDAC with TrueBasic}

The TrueBasic programming environment is easy to use and relatively powerful. Most features of TrueBasic are familiar to anyone who has used any modern version of Basic, but several features deserve note. TrueBasic programs are portable from one system to another, so programs can be written on one system and used on another. TrueBasic also has its own editor for writing programs. Simple graphics can be used in programs, and there is a "get mouse" command to track mouse actions. The graphic and mouse functions allow programs to accept and interpret mouse clicks on graphically defined parts of the monitor. Finally, TrueBasic has a Communications Support package of subroutines that interfaces the Basic programming environment to peripheral hardware through the serial port. TrueBasic has limitations in comparison with Walter and Palya's (1984) ECBasic, as we will note later. Table 1 shows some of the TrueBasic code that runs an operant chamber. Only the lines that illustrate advantages or disadvantages of this system are included. Line numbers, optional in TrueBasic, are included only for expository purposes.

Line 20 calls the communications package library, and line 30 opens an 8-bit, 9600 -baud link to the IDAC interface through the serial port. TrueBasic permits the 


\begin{tabular}{|c|c|c|c|c|c|c|c|c|c|c|c|c|}
\hline & A & B & c & D & K & $\mathbf{L}$ & $\mathbf{s}$ & $T$ & U & v & $\bar{w}$ & $\bar{x}$ \\
\hline 1 & \multirow{2}{*}{\multicolumn{12}{|c|}{\begin{tabular}{|l|l|l|} 
MACONTROL - APPLICATION TEMPLATE \\
S Dur
\end{tabular}}} \\
\hline 2 & & $\mathrm{~s}$ & & & & Dur & & & & & & \\
\hline & & & & & & & & & & & & \\
\hline & & $\mathrm{S}$ & & & & & & & & & & \\
\hline 23 & ANALOC & $E$ & Outp & & & & & & Bran & & & \\
\hline 24 & OUTPज़ & $Q$ & Val & & & & & & to: & & & \\
\hline 25 & $\mathrm{CH} \# 00$ & & & & & & & & & & & \\
\hline 26 & $\mathrm{CH} 01$ & & & & & & & & & & & \\
\hline 27 & & $\mathrm{~S}$ & & & & Dur & & & & & & \\
\hline 28 & & $E$ & & On/Ofi & of & betw & & & & & Timer & Branch \\
\hline 29 & DIGITAL & $Q$ & $1 / 0$ & $(1,0)$ & Samp & Samp & Criteria & $\mathrm{BOL}$ & $=$ & $\mathrm{BOH}$ & (minutes & to: \\
\hline 30 & & & & & & & & & & & & \\
\hline 31 & $\mathrm{CH} \# 00$ & 2 & 0 & P & & & & & 3 & & & \\
\hline 32 & $\mathrm{CH} \# 01$ & & & & & & & & & & & \\
\hline 33 & $\mathrm{CH} \# 02$ & & & & & & & & & & & \\
\hline 34 & $\mathrm{CH} \# 03$ & & & & & & & & & & & \\
\hline 35 & $\mathrm{CH} \# 04$ & & & & & & & & & & & \\
\hline 36 & $\mathrm{CH} \# 05$ & & & & & & & & & & & \\
\hline 37 & $\mathrm{CH} \# 06$ & & & & & & & & & & & \\
\hline 38 & $\mathrm{CH} \# 07$ & & & & & & & & & & & \\
\hline 39 & $\mathrm{CH} \# 08$ & 3 & & & C & & & & & & 1 & 4 \\
\hline 40 & $\mathrm{CH} \# 08$ & 4 & & & c & & & & & & 1 & 1 \\
\hline 41 & $\mathrm{CH} \# 08$ & 1 & & & C & & 1 & & 2 & & & \\
\hline 42 & $\mathrm{CH} \# 11$ & & & & & & & & & & & \\
\hline 43 & $\mathrm{CH} \# 12$ & & & & & & & & & & & \\
\hline 44 & $\mathrm{CH} \# 13$ & & & & & & & & & & & \\
\hline 45 & $\mathrm{CH} \# 14$ & & & & & & & & & & & \\
\hline 46 & $\mathrm{CH} \# 15$ & & & & & & & & & & & \\
\hline
\end{tabular}

Figure 3. IDAC's application template for an FI 2-min operant schedule.

opening of different "screens" so that graphic, print, and mouse input functions can be confined to a separate portion of the computer screen. Lines $50-70$, for example, create a screen window on the middle right section of the computer monitor to toggle extinction with mouse clicks (see lines 250-290).

This program runs ratio and interval schedules and asks for both ratio and time requirements on lines 90 and 100. Thus, a ratio 40 schedule would set fratio at 40 and ftimemin at 0 . A 60 -sec interval schedule would set ftimemin at 60 and fratio at 0 . Whether the schedule is fixed or variable is set by varl on Line 110 .

The IDAC system has a library of read and write commands that are sent back and forth as string variables in send and receive commands, as can be seen in lines 130-170. In the AS variable on line 130 , A0 specifies ASCII protocol, 0 is the default IDAC identification number, and RD BT $(5,0)$ reads bit 0 of input byte 5 on the IDAC data bus. Bit 0 of byte 5 has a value of 0 or 1 depending on the presence or absence of a response input. IDAC will not store inputs, so the program must look for inputs when they are most likely. B\$ of line 160 returns information from IDAC in the form !1,0,0, where the 1 is an identification number, the second character is 0 or $\mathrm{E}$ (for error in transmission), and the third character is the status of the bit $(0$ or 1$)$.
For those interested in other IDAC commands, Table 2 lists all the digital and analog read and write commands.

The only peripheral response shaping that $I$ do is to have inputs trigger a $300-\mathrm{msec}$ electromagnetic pulse to the IDAC input relays. Inputs less than this duration were occasionally missed by the TrueBasic program. With minimal response shaping, my software must be alert to transmission bugs. Lines 140 and 170 perform a two step "for-next" check for inputs, to be sure that all inputs are detected. An occasional scrambling of the B \$ input from IDAC would halt the program with a TrueBasic error (that is, if $\mathrm{B} \$$ was returned in a form different than expected), especially after food pellets were delivered. Lines 190-230 provide an error trapping loop for scrambled inputs. The way in which TrueBasic error trapping works is that an error generated anywhere in the loop executes commands that follow "use" on 220 . I list no commands, which means that TrueBasic errors generated during input detection are ignored. Line 200 detects IDAC error messages that appear in the fourth character from the B \$ string. In several years of using IDAC, I have never seen such an error.

Response inputs are caught on line 210 and direct program flow to a response evaluation subroutine that begins on line 310 . Lines $250-290$ check for mouse inputs, which toggle extinction. 


\begin{tabular}{|c|c|c|c|c|c|c|c|}
\hline & $\bar{A}$ & $\bar{B}$ & C & $\bar{D}$ & $\bar{E}$ & $\bar{F}$ & $\overline{\mathbf{G}}$ \\
\hline 1 & & \#\#\# & $\# \# \# \#$ & & & & \\
\hline 2 & & & & & & & \\
\hline 3 & START DAT & \#\#\# & START TIME & \#\#\#\# & 38520 & & \\
\hline 4 & $\operatorname{TICS}(1 / 60 \mathrm{~S}$ & SINCE SYST & 6482 & & & & \\
\hline 5 & & & & & & & \\
\hline 6 & PROCESS \# & 1 & & & & & \\
\hline 7 & & & & & & & \\
\hline 8 & $\mathrm{CH} \#$ & SEO & SEO & MEAN/ & NUMBER & STANDARD & LAST \\
\hline 9 & & NMM & TYPE & PUSES & READINGS & DEVIATION & $\begin{array}{l}\text { VALUE } \\
\end{array}$ \\
\hline 10 & $0-8$ & 1 & D & 1 & & & \\
\hline 11 & $0-0$ & 2 & $\infty$ & & & & \\
\hline 12 & $0-8$ & 3 & D & 10 & & & \\
\hline 13 & $0-8$ & 4 & D & 13 & & & \\
\hline 14 & $0-8$ & 1 & $\bar{D}$ & 1 & & & \\
\hline 15 & 0.0 & 2 & $\infty$ & & & & \\
\hline 16 & $0-8$ & 3 & D & 14 & & & \\
\hline 17 & $0-8$ & 4 & $\bar{D}$ & 11 & & & \\
\hline 18 & 0.8 & 1 & D & 1 & & & \\
\hline 19 & 0.0 & 2 & $D O$ & & & & \\
\hline 20 & $0-8$ & 3 & D & 12 & & & \\
\hline 21 & $0-8$ & 4 & D & 17 & & & \\
\hline 22 & $0-8$ & 1 & $\bar{D}$ & 1 & & & \\
\hline 23 & $0-0$ & 2 & $D$ & & & & \\
\hline 24 & 0.8 & 3 & $\bar{D}$ & 11 & & & \\
\hline 25 & $0-8$ & 4 & D & 12 & & & \\
\hline
\end{tabular}

Figure 4. IDAC's LOG report for the FI 2-min experiment.

Once an input is detected and the response evaluation subroutine is activated, another subroutine (not shown) waits until the response is over so as not to count the same input twice. This produces a delay of up to $300 \mathrm{msec}$, the duration of all inputs. This delay limits the number of input and output transmissions that can occur in a time period, but it has never hindered data collection in routine operant experiments.

Line 320 puts the time of the current response in a data matrix, and 330 counts the number of responses since the last reinforcement. Line 350 counts the number of responses that fulfill a ratio requirement, and 360 gets the time of the response with TrueBasic's time function. TrueBasic times accumulate independently of program steps, and they are expressed as seconds since midnight on the Macintosh computer. The times are accurate to $.1 \mathrm{sec}$.

If the number of responses is fewer than the ratio requirement (line 380 ), or the minimum time delay since the last reinforcement has not expired (line 400), the subroutine is exited and no reinforcement is given.

Lines 420-460 deliver a food pellet with IDAC's Write Byte (WR BY) command. Separate on and off commands are used because TrueBasic has no pulse command. Although most pellet dispensers operate with a recommended 50-msec pulse, I have found that a 100 msec pulse works more reliably, but I do not know whether this is due to the TrueBasic code or to the dispenser itself.

Once a reinforcement is delivered, lines 480-510 determine the next ratio or interval requirement. On 500 , ratio will be the minimum number of barpresses for the next reinforcement. If a ratio schedule is fixed, varl equals 0 , and ratio simply equals fratio, the value set at run time. (For ratio schedules, timemin and ftimemin are irrelevant, because they were set to zero at run time. Conversely, all ratio requirements are irrelevant for interval schedules.)

For a variable ratio schedule, ratios will vary between 0 and twice the value of fratio. Rnd generates a random number between 0 and 1 , and the expression $(2 *(r-$ $.5))^{*}$ varl yields a number between -1 and +1 . Thus, a variable ratio 20 will yield ratio requirements between 0 and 40 . The same rules apply to time minimums for interval schedules (line 510).

On subsequent lines not shown, the times of all responses since the last reinforcement are written to the data file. Writing data to disk occurs immediately after a reinforcement is delivered, so as not to compromise the ability of the program to detect inputs. By way of comparison, MaControl software writes data to disk after a fixed number of responses. MaControl's default is 100 responses, but is changeable at run time. By writing to disk after a certain number of inputs, MaControl will miss inputs at these times.

I write a variety of information to disk, including the status of stimulus lights, the ratio and interval requirements, and so forth, but the most important data are barpress times read to a data matrix on line 320 . Times of all responses since the last reinforcement collect here until 
data are written to disk and the matrix cleared. This means that the data matrix must be dimensionalized to handle the maximum number of response inputs expected between successive reinforcements. The data are written as a TrueBasic text file, which is later opened by Microsoft Excel as a spreadsheet. To make successive units of data appear in adjacent spreadsheet cells, chr\$( 9$)$ delimiters are inserted between successive data (otherwise, all the data will end up in one spreadsheet cell).

\section{Using Microsoft Excel to Make Cumulative Graphs}

To graph the data, we later open the TrueBasic text file from Microsoft Excel and use a macro whose highlights appear below. Figure 5 shows the spreadsheet organiza-

Table 1

TrueBasic Controlling Program

20 library "Comlib*"

30 call Com_open (\#1,1,9600,"D8 P-") ! channel to idac

50 Open \#7:screen $.5,1,5, .7$

60 print

70 print “ EXTINCTION TOGGLE”

90 input prompt "Ratio [0 if Int] ?":fratio

100 input prompt "Time interval [0 if ratio] ?":ftimemin

110 input prompt "Fixed $(0)$ or varied $(1)$ ?":varl

130 let $\mathrm{A} \$=" \$ \mathrm{~A} 00 \mathrm{RD}$ BT $(5,0) "$

140 for $\mathrm{i}=1$ to 2

150 call Send_cr(AS)

160 call Receive(BS)

170 next $\mathrm{i}$

190 when error in

200 if $B \$(4: 4)=" E "$ then call error

210 if $\operatorname{val}(B \$(6: 6))>0$ then call response( )

220 use

230 end when

250 get mouse $x, y, z$

260 if $z=3$ and $x>1$ and $y<0$ and $y>-.6$ then

270 let ext $=$ ext +1

280 else

290 end if

310 sub response()

320 let data(count $1+1,1)=$ time

330 let count $1=$ count $1+1$

350 let count $2=$ count $2+1$

360 let currenttime $=$ time

380 if count $2<$ ratio then exit sub

390 let timediff $=$ (currenttime-oldtime)

400 if timediff $<$ timemin then exit sub

420 let $A \$=" \$ A 00$ WR BY $(4,1) "$

430 call Send_cr(A\$)

440 pause 1

450 let a $\$=" \$ A 00$ WR BY $(4,0) "$

460 call Send_cr(A\$)

480 let oldtime $=$ currenttime

490 let $r=$ rnd

500 let ratio $=$ fratio + fratio $*(2 *(\mathrm{r}-.5))^{*}$ var 1

510 let timemin $=$ ftimemin+ftimemin $*(2 *(r-.5))^{*}$ var 1
Table 2

WAC Read and Write Commands

$\begin{array}{ll}\text { Commands for Digital Transmission } \\ \text { \$A0 id RD BT }(\mathrm{a}, \mathrm{b}) & \text { reads bit b of byte a } \\ \text { \$A0 id RD BY }(\mathrm{a}) & \text { reads byte a } \\ \text { \$A0 id RD WD }(\mathrm{a}) & \text { reads a word of data } \\ \text { \$A0 id WR BS }(\mathrm{a}, \mathrm{b}) & \text { sets bit b of byte a to } 1 \\ \text { \$A0 id WR BC }(\mathrm{a}, \mathrm{b}) & \text { sets bit b of byte a to 0 } \\ \text { \$A0 id WR BY }(\mathrm{a}, \mathrm{d}) & \text { sets byte a to value d } \\ \text { \$A0 id WR WD }(\mathrm{a}, \mathrm{dd}) & \text { sets byte a to word dd } \\ \text { Commands for Analog Transmission } \\ \text { \$A0 id RD AN }(\mathrm{a}, \mathrm{c}) & \text { reads analog channel c of byte a } \\ \text { \$A0 id WR AN }(\mathrm{a}, \mathrm{dd}) & \text { writes output data to channel a }\end{array}$

tion of data from a VI 30 -sec schedule. Column $\mathrm{A}$ has raw response times, and columns B-G contain ancillary information. Column $\mathrm{C}$ flags stimulus light status, and column $\mathrm{D}$ flags reinforcements. $\mathrm{E}$ and $\mathrm{F}$ note the ratio and time requirements just met when reinforcement was delivered.

My macro puts in column $\mathrm{H}$ the time, in minutes, of every response, using the first response as minute zero. Thus the second barpress occurs at minute (52675.3 $52669.9) / 60$, or $.09 \mathrm{~min}$. Translating the actual times of successive barpresses into the number of barpresses per unit time was not a trivial programming problem. As a first step, my macro uses Excel's DATA.SERIES command to number the responses in column I. The macro then places Excel's LOOKUP command in column J, and the LOOKUP figures cumulative response per minute. The form of the command is LOOKUP (X, Vector1, Vector2). The variable $X$ represents the time in minutes. For my undergraduate labs, experiments include $40 \mathrm{~min}$ of data collection, so $X$ loops from 1 to 40 as seen below. Vectorl corresponds to the barpress times in column $\mathrm{H}$, and Vector2 the consecutively numbered barpresses in column I. LOOKUP searches Vectorl for the largest number still smaller than $\mathrm{X}$, and returns the corresponding number of Vector2.

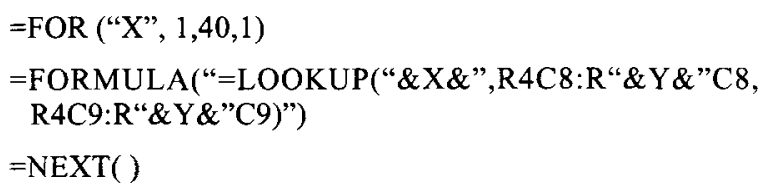

As an example, to figure the number of barpresses in the first minute, $\mathrm{X}$ equals one and LOOKUP searches column $\mathrm{H}$ for the largest value less than one, this being .492 in row 12 . LOOKUP returns the corresponding number of barpresses, 9 , from column $I$. When $X$ is two, the largest value in column $\mathrm{H}$ less than $2 \mathrm{~min}$ is at minute 1.965 . The corresponding value in column $\mathrm{I}$ is 28. In the LOOKUP command the $\mathrm{Y}$ variable specifies the vector size in the spreadsheet, which is determined by other macro commands. My actual LOOKUP formulas get a bit more complex because I reset the cumulative response to zero whenever a value of 200 is ex- 
ceeded. The macro then graphs cumulative responses. Figure 6 is an example from a VI 30-sec schedule.

Making cumulative graphs with a finer grain (that is, with time units smaller than $1 \mathrm{~min}$ ) is possible. One could have the For-Next loop in the macro increment with a step value of .5 (for $30 \mathrm{sec}$ ) or .1 (for $6 \mathrm{sec}$ ), rather than in step value of 1 , as I have done.

\section{Comparison With the Walter and Palya System}

Walter and Palya (1984) described a powerful interface system for the Macintosh that can operate in standard laboratory situations. Improvements and modifications in their system have been reported as well (Palya \& Walter, 1993; Weisman \& Palya, 1988). Their system is more ambitious in the sense that they have developed their own programming language (ECBasic) and an operating environment that can connect several interfaces to a single host controller.

Comparing the languages, ECBasic is designed specifically for experimental control, and contains several advantageous features. For instance, their time function is accurate to $.01 \mathrm{sec}$, and, like TrueBasic's, is independent of program steps. They also have a PULSON ( ) command to pulse on peripheral equipment, whereas I must pulse with two commands. Another interesting feature is their CALL INPUT $(\mathrm{X}, \mathrm{R})$, which checks for a count of response inputs that accumulates independently of program code.

The IDAC system as it is described here may have advantages for smaller labs with stand-alone stations, since it is simpler and it requires little technical expertise. The interface comes as a complete package with input and output relay panels. I run IDAC interfaces with Macintosh Plus and SEs discarded by colleagues as they have upgraded to newer systems. Many persons already have some mastery of Basic, or can learn it easily. IDAC also comes with its own control software, which can be adequate for some users. Finally, the fact that IDAC handles analog $\mathrm{I} / \mathrm{O}$ means that it can be used for applications other than those described here. This system may be a good solution for those seeking a general, all-purpose laboratory interface.

\begin{tabular}{|c|c|c|c|c|c|c|c|c|c|c|c|}
\hline & $\mathbf{A}$ & $\mathbf{B}$ & C & D & $\mathbf{E}$ & $\mathbf{F}$ & $\mathbf{G}$ & $\mathrm{H}$ & 1 & $J$ & $\mathbf{K}$ \\
\hline 1 & ratio $=1$ time & $3 \min =3$ & & & & & & & & & \\
\hline 2 & & & & & & & & & & & \\
\hline 3 & Time & And? & ? status & $S(r)$ & Ratio & Interval & Ext? & $\operatorname{time}$ & & $\mathbf{R}$ & \\
\hline 4 & 52669.9 & 0 & 1. & 1 & 0 & 0 & 0 & & 1 & 9 & \\
\hline 5 & 52675.3 & 0 & 1 & 0 & 0 & 0 & 0 & 0.09 & 2 & 28 & \\
\hline 6 & 52678.5 & 0 & 1 & 0 & 0 & 0 & 0 & 0.143 & 3 & 44 & \\
\hline 7 & 52681.5 & 0 & 1 & 0. & 0 & 0 & 0 & 0.193 & 4 & 68 & \\
\hline 8 & 52684.7 & 0 & 1 & 0 & 0 & 0 & 0 & 0.247 & 5 & 90 & \\
\hline 9 & 52686.5 & 0 & 1 & 0 & 0 & 0 & 0 & 0.277 & 6. & 110 & \\
\hline 10 & 52690.2 & 0 & 1 & 0 & 0 & 0 & 0 & 0.338 & 7 & 140 & \\
\hline 11 & 52695.8 & 0 & 1 & 0 & 0 & 0 & 0 & 0.432 & 8 & 168 & \\
\hline 12 & 52699.4 & 0 & 1 & 0 & 0 & 0 & 0 & 0.492 & 9 & 190 & \\
\hline 13 & 52746.6 & 0 & 1 & 1 & 0 & 52 & 0 & 1.278 & 10 & 6 & \\
\hline 14 & 52755.8 & 0 & 1 & 1. & 0 & 8 & 0 & 1.432 & 11 & 34 & \\
\hline 15 & 52760.2 & 0 & 1 & 0 & 0 & 0 & 0 & 1.505 & 12 & 55 & \\
\hline 16 & 52762.1 & 0 & 1 & 0 & 0 & 0 & 0 & 1.537 & 13 & 66 & \\
\hline 17 & 52763.3 & 0 & 1 & 0 & 0 & 0 & 0 & 1.557 & 14 & 95 & \\
\hline 18 & 52764.2 & 0 & 1 & 0 & 0 & 으. & 0 & 1.572 & 15 & 130 & \\
\hline 19 & 52765.8 & 0 & 1 & 0 & 0 & 0 & 0 & 1.598 & 16 & 152 & \\
\hline 20 & 52766.7 & 0 & 1 & 0 & 0 & 0 & 0 & 1.613 & 17 & 162 & \\
\hline 21 & 52767.5 & 0 & 1 & 0 & 0 & 0 & 0 & 1.627 & 18 & 176 & \\
\hline 22 & 52770.2 & 0 & 1 & 0 & 0 & 0 & 0 & 1.672 & 19 & 189 & \\
\hline 23 & 52771 & 0 & 1 & 0 & 0 & 0 & 0 & 1.685 & 20 & 10 & \\
\hline 24 & 52773.5 & 0 & 1 & 0 & 0 & 0 & 0 & 1.727 & 21 & 25 & \\
\hline 25 & 52775.2 & 0 & 1 & 0 & 0 & 0 & 0 & 1.755 & 22 & 34 & \\
\hline 26 & 52779.6 & 0 & 1 & 0 & 0 & 0 & 0 & 1.828 & 23 & 62 & \\
\hline 27 & 52781.5 & 0 & 1 & 0 & 0 & 0 & 0 & 1.86 & 24 & 92 & \\
\hline 28 & 52783.8 & 0 & 1 & 0 & 0 & 0 & 0 & 1.898 & 25 & 126 & \\
\hline 29 & 52784.7 & 0 & 1 & 0 & 0 & 0 & 0 & 1.913 & 26 & 149 & \\
\hline 30 & 52786.8 & 0 & 1 & 0 & 0 & 0 & D) & 1.948 & 27 & 171 & \\
\hline 31 & 52787.8 & 0 & 1 & 1 & 0 & 29 & 0 & 1.965 & 28 & 199 & \\
\hline 32 & 52790.6 & 0 & 1 & 0 & 0 & 0 & 0 & 2.012 & 29 & 12 & \\
\hline 33 & 52812 & 0 . & 1 & 1 & 0 & 20 & 0 & 2.368 & 30 & 42 & \\
\hline 34 & 52819.6 & 0 & 1 & 0 & 0 & 0 & 0 & 2.495 & 31 & 72 & \\
\hline
\end{tabular}

Figure 5. Response data summarized by the macro. 
Student | VI 30 sec | 3 June 1993

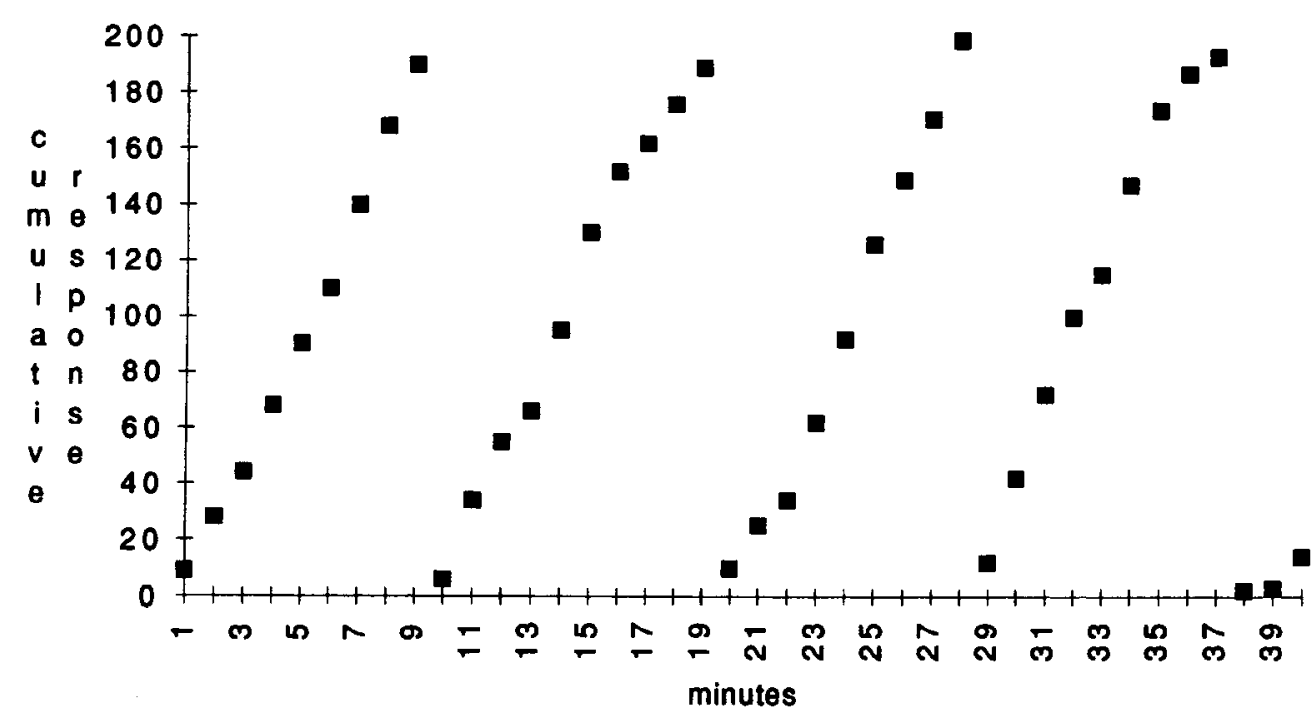

Figure 6. Response data as a cumulative graph.

\section{REFERENCES}

Bates, T. C. (1991). A Macintosh II psychophysiology system. Behavior Research Methods, Instruments, \& Computers, 23, 395-402.

Costin, D. (1988). MacLab: A Macintosh system for psychology labs. Behavior Research Methods, Instruments, \& Computers, 20, 197200.

Hatten, J. L., \& Hatten, A. D. (1989). Implementing an undergraduate research apprenticeship with the Macintosh computer. Behavior Research Methods, Instruments, \& Computers, 21, 142-147.

Palya, W. L., \& Walter, D. E. (1993). A powerful, inexpensive experiment controller for IBM PC interface and experiment control language. Behavior Research Methods, Instruments, \& Computers, 25, 127-136.
SCHNEIDER, W. (1991). Equipment is cheap, but the field must develop and support common sof tware for psychological research. Behavior Research Methods, Instruments, \& Computers, 23, 114-116.

WAlter, D. E., \& Palya, W. L. (1984). An inexpensive experiment controller for stand-alone applications or distributed processing networks. Behavior Research Methods, Instruments, \& Computers, 16, 125-134.

Weisman, R., \& Palya, W. L. (1988). Development and operating environments for a network of Walter/Palya experiment controllers on the Macintosh computer. Behavior Research Methods, Instruments, \& Computers, 20, 93-96.

(Manuscript received January 18, 1994; revision accepted for publication June 23, 1994.) 